\title{
Multichannel Data Compression using Wavelet Subbands Arranging Technique
}

\author{
Ershad Sharifahmadian, Yoonsuk Choi, Shahram Latifi \\ Department of Electrical \& Computer Engineering \\ University of Nevada, Las Vegas \\ 4505 Maryland Parkway \\ Las Vegas, NV 89154-4026
}

\begin{abstract}
To reduce the amount of data and preserve necessary signal quality for multichannel data transmission in many applications such as meteorology, or telemedicine, a new technique called WSAT is presented. The proposed technique is designed to deal with the large amount of multichannel data for transmission, and real-time analysis. The proposed approach has exact control on the bit rate in order to achieve the required quality. For different applications, the proposed method is tested. For telemedicine, the method is employed on selected records from the MIT-BIH arrhythmia database. For meteorology, climate data from Nevada climate change database is utilized. From the obtained results, it is concluded that the proposed technique is an appropriate approach to simultaneously compress multichannel data with significant low compressed data rate at low error. As an example, APRD values for multichannel ECG compression is mostly less than $5 \%$ which is recommended by the American Heart Association for routine visual readings of compressed and reconstructed ECG signals.
\end{abstract}

\section{Keywords}

Meteorology, Multichannel compression, Telemedicine, Wavelet, WSAT.

\section{INTRODUCTION}

To provide a better performing system for transmitted, distributed, or processed data, data compression is emphasized. Data compression improves the system performance and reduces redundancy in stored data.

With enough information about data's characteristics including the type, size, and statistical characteristics of data, it is possible to define or choose an appropriate method for data compression.

In general, to achieve a high compression ratio in multichannel data compression, it is necessary to reduce redundancy between adjacent data of every channel of multichannel data acquisition system.

In this study, Wavelet Subbands Arranging Technique (WSAT) is proposed to simultaneously compress multichannel data. The proposed method can be applied in different areas. Here, the application of the method is illustrated and explained for meteorology and telemedicine.

\subsection{Telemedicine}

ECG monitoring and recording facilities produce huge amount of data, and those data should be transmitted, stored and processed in reasonable amounts of time. Thus, it is necessary to efficiently compress data.

To provide higher compression ratio and better signal quality without affecting the diagnostic characteristics of the reconstructed data, ECG compression methods have been developed. There are different types of compression methods have been reported for single channel ECG compression in the literature [2]-[6]. Most of the compression techniques have been developed for single channel ECG compression. Although some of those methods were applied on multichannel ECG signals to individually compress ECG data, however, few methods can deal with the problem of multichannel ECG compression simultaneously.

ECG signals are non-stationary, each beat cycle includes mainly of QRS complex, P, T, S-T segment, baseline, etc. The first three parts are most useful in clinical diagnosis. Whereas the major energy of the $\mathrm{P}$ and $\mathrm{T}$ waves place below $5 \mathrm{~Hz}$, and the major energy of the QRS complex places between 5-15 Hz, the type of filter bank and the number of wavelet decomposition levels should be appropriately chosen [7]. The biorthogonal 9/7 tap filters are suitable choice for ECG compression and image compression [8].

Moreover, the number of decomposition levels will be set at least four for ECG compression.

The energy of a signal is typically placed in the lower frequencies. For physiological signals such ECG, the higher frequency content is almost close to zero. Therefore, if lower frequency content is kept, lots of insignificant data are dismissed.

\subsection{Meteorology}

Typically, the scientific climate data is voluminous and there is a high traffic to/from climate data portal by the scientific community, research groups, and general readers. It is not always possible to increase bandwidth to support all clients, and provide good service for on line users. One solution is efficiently compressing climate data.

\section{PROPOSED METHOD}

First, the wavelet transform is applied on data. During the wavelet decomposition, the initial data is passed through a low pass filter and a high pass filter. Then, two subbands including approximation part $(A)$ and detail part $(D)$ are provided after down-sampling of the two filtered outputs.

If the $K$-level wavelet decomposition is done, $K+l$ wavelet coefficient subbands are provided.

The length of the approximation part is called $L_{A}$ and it is equal to the length of the detail part.

Now, the threshold of each wavelet subband is defined. $T h r_{k}$ which is the threshold of the $k^{\text {th }}$ wavelet subband is formulated as follows:

$T h r_{k}=2^{\left\lfloor\log _{2}\left(\operatorname{Max}_{i \in k \text { th subband }}\left|w_{i}\right|\right)\right\rfloor}$ 
where $w_{i}$ is the wavelet coefficient at location (i) [9]. Among the calculated thresholds, the largest threshold is chosen as the initial operation threshold.

Wavelet coefficients with larger magnitude are generally concentrated in lower frequency wavelet subbands [10]. Arranged wavelet subbands of input channels cause more efficient multichannel compression, because appropriate parentoffspring relations among coefficients of wavelet subbands are formed at a hierarchical structure.

In order to simultaneously compress multichannel signals, first, the subbands of channels are appropriately arranged. To arrange the wavelet subbands, the largest threshold from each input channel is calculated based on equation (1). Among input channels, a channel with the largest threshold is chosen (initial channel). The second chosen channel has threshold larger than residual channels but its threshold is smaller than that of the initial channel. Rest of channels will be selected by this procedure. Therefore, a new structure is formed in which the approximation and detail subbands of selected channels are rearranged and sorted from highest subband threshold to lowest subband threshold.
Now, the wavelet coefficients are rearranged in hierarchies, with roots placed in the lowest wavelet frequency subband, and branching into higher wavelet frequency subbands consecutively.

In the hierarchical structure, $D(i)$ is defined as the set of all descendants of a node defined at position $(i)$, which is called a set of type I. $E(i)$ is defined as the set of all descendants excluding offspring of a node defined at position $(i)$, which is called a set of type II.

After selection of the initial operation threshold, the given threshold is compared with the threshold of every subband. This process is done from the highest frequency subband to the lowest frequency subband, and the process will be stopped if the threshold of a subband becomes equal to the initial operation threshold for first time. Then, the position of the last coefficient from the wavelet subband where comparison was performed up to, is recorded. The given position (called as frontier) restricts evaluation by which the significance of $E(i)$ or $D(i)$ is determined [9].

During compression process, the main information about the wavelet coefficients is categorized as follows:

C1: The category of positions relating to wavelet coefficients that have magnitude smaller than a given threshold.

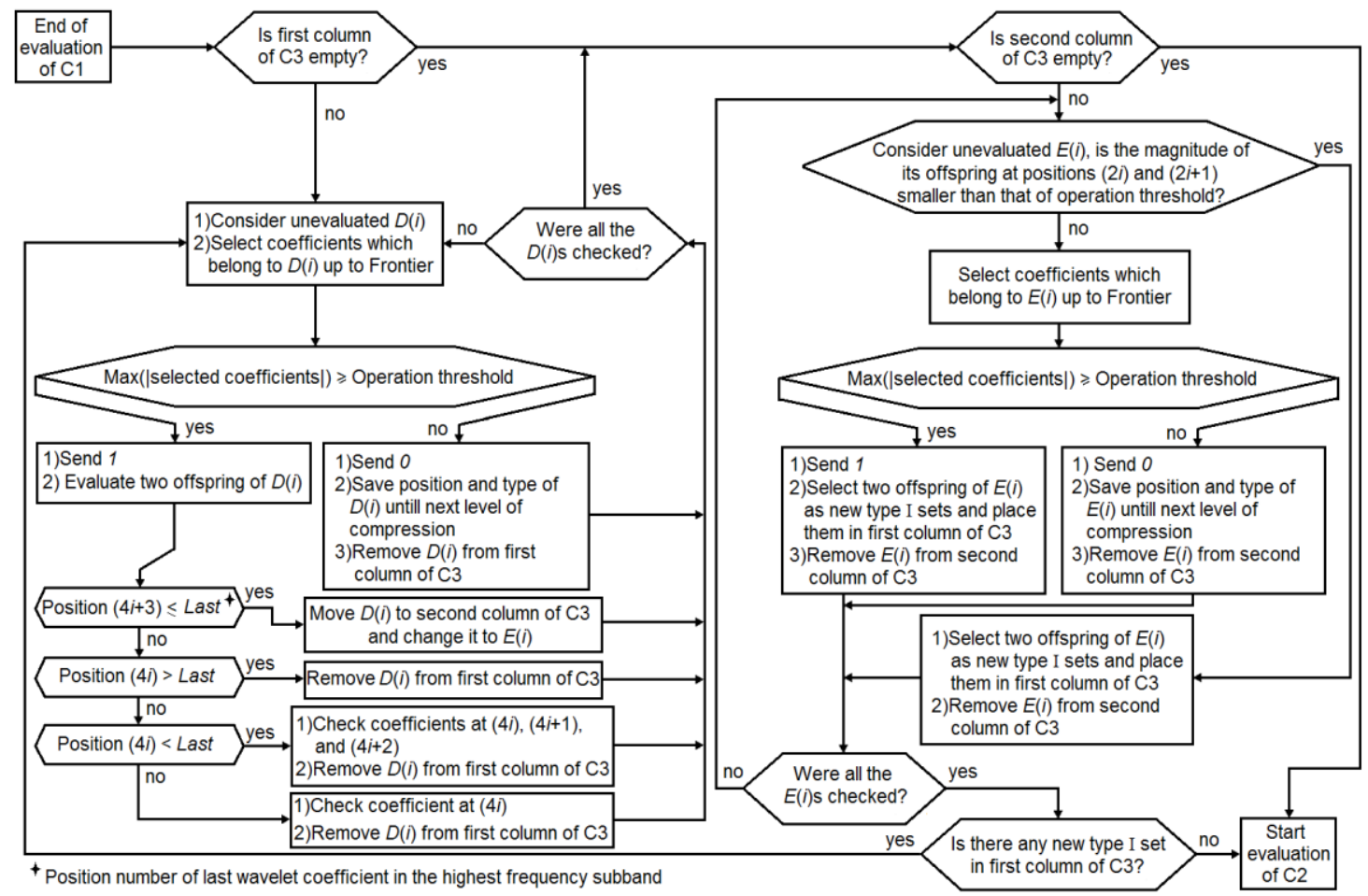

Figure 1. Procedure of evaluation of $\mathrm{C3}$

C2: The category of positions relating to wavelet coefficients that have magnitude larger than (or equals to) a given threshold.

C3: A two-column matrix including the category of positions corresponding to the sets of the wavelet coefficients defined by hierarchical structure; those sets have magnitude smaller than a given threshold.

The wavelet coefficients are encoded in various scans of the coefficients. During each scan, the coefficients with magnitudes which are larger than a certain threshold are encoded.

At first, the $\mathrm{C} 2$ is set as empty. If the approximation subband (i.e. $\left.L_{A}\right)$ is even, the positions of $\left\{(0)\right.$ to $\left.\left(L_{A}-1\right)\right\}$ will be placed into the $\mathrm{C} 1$, and the positions of $\left\{\left(L_{A} / 2\right)\right.$ to $\left.\left(L_{A}-1\right)\right\}$, will be placed into the $\mathrm{C} 3$ as type $\mathrm{I}$. If the approximation subband is odd, the positions of $\left\{(0)\right.$ to $\left.\left(L_{A}-2\right)\right\}$ will be placed into the $\mathrm{C}$, and the positions of $\left\{\left(L_{A}-1 / 2\right)\right.$ to $\left.\left(L_{A}-1\right)\right\}$, will be placed into the $\mathrm{C} 3$ as type $\mathrm{I}$.

A wavelet coefficient relating to the $\mathrm{C} 1$ is significant if the coefficient is larger than (or equal to) the current operation threshold. In this situation, a one is sent with a sign bit, and its position moved to the $\mathrm{C} 2$. If the coefficient is not significant, a zero is sent and ts position kept until the next compression 
level. For the negative sign, the sign bit is set as 0 , otherwise the sign bit is set as 1 .

After checking the $\mathrm{C} 1$, wavelet coefficients relating to the $\mathrm{C} 3$ are checked. For a $D(i)$, the following process is considered (the position number of last wavelet coefficient in the highest frequency subband is named as "Last"):

if $D(i)$ is significant

Send 1 .

Evaluate two offspring of $D(i)$ like evaluation of

coefficients related to $\mathrm{C} 1$.

if position number $(4 i+3) \leq$ Last

Move $D(i)$ to second column of $\mathrm{C} 3$ and

change it to $E(i)$.

elseif position number $(4 i)>$ Last

Remove $D(i)$ from first column of $\mathrm{C} 3$.

elseif position number $(4 i)<$ Last

Check coefficients at positions $(4 i),(4 i+1)$,

and $(4 i+2)$

Remove $D(i)$ from first column of $\mathrm{C} 3$.

else

Check coefficient at position (4i)

Remove $D(i)$ from first column of $\mathrm{C} 3$. end

else

Send 0

Save position and type of $D(i)$ until next

compression level.

Remove $D(i)$ from first column of C3.

end

When all sets in the first column of the $\mathrm{C} 3$ are evaluated, $E(i)$ in the second column of the $\mathrm{C} 3$ are evaluated.

Before evaluation of each $E(i)$, the two offspring, direct descendants of a wavelet coefficient at position (i), are emphasized. These direct children of a tree node at location $(i)$ are utilized to improve the evaluation of $E(i)$ s. If these direct children are smaller than the current operation threshold, they are chosen as new entries of type I, and directly placed in the first column of the C3. Therefore, evaluation of $E(i)$ is not performed. If at least one of the direct children is larger than (or equal to) the current operation threshold, evaluation of $E(i)$ is performed. In this case, if $E(i)$ is not significant, a zero is sent. The $E(i)$ is then removed from the second column of the $\mathrm{C} 3$, its type and position are kept until the next compression level.

If the set of type II is significant, a one is sent. Its two offspring are then selected as new sets of type I and placed in the first column of the $\mathrm{C} 3$. In next, the $E(i)$ is removed from the second column of the $\mathrm{C} 3$.

When all $E(i) \mathrm{s}$ in the second column of the $\mathrm{C} 3$ were evaluated, new $D(i)$ s in the first column of the $\mathrm{C} 3$ are evaluated. The evaluation of the $\mathrm{C} 3$ is completed when no new $D(i)$ placed in the first column of the $\mathrm{C} 3$. The procedure of evaluation of the $\mathrm{C} 3$ is shown in Figure 1

After the evaluation of the $\mathrm{C} 3$, evaluation of $\mathrm{C} 2$ is started. Every old entry of the $\mathrm{C} 2$ is checked. When an old entry of the $\mathrm{C} 2$ is significant over the current operation threshold, a zero is sent, otherwise a one is sent.

After evaluation of all entries in the $\mathrm{C} 2$, the magnitude of all coefficients for which their positions placed in the $\mathrm{C} 2$ and are larger than the current operation threshold, are subtracted by the current operation threshold.

After the evaluation of the $\mathrm{C} 2$, the current operation threshold is halved, the evaluation of $\mathrm{C} 1, \mathrm{C} 3$, and $\mathrm{C} 2$ are repeated as far as the desirable bit rate is met.

\section{RESULTS}

To test the redundancy removing ability of compression methods, the compression ratio (CR) is typically used. The $\mathrm{CR}$ is dependent on sample resolution, therefore, its value could change with different digitization specifications. A more suitable evaluation criterion for efficiency of a compression technique is the compressed data rate or CDR (bits/s or bps) [11]-[12]. The percent root mean square difference (PRD) is utilized as the evaluation measure for the fidelity of reconstructed data. The evaluation measures are formulated as follows:

$$
C R=\frac{s r \times L}{B_{\text {compression }}}
$$

And

$C D R=\frac{R_{s} \times B_{\text {compressia }}}{L}$

And

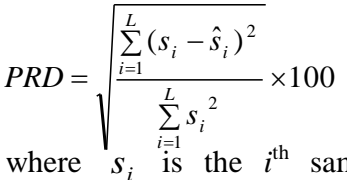

where $s_{i}$ is the $i^{\text {th }}$ sample from original data, $\hat{s}_{i}$ is its reconstructed sample, $s r$ is the sample resolution (bits/sample), $B_{\text {compressin }}$ is the total number of bits to be transmitted or stored after compression, $L$ is the number of samples in the original signal, and $R_{s}$ is the sampling rate (samples/sec). The average percent root mean square difference (APRD) is utilized to evaluate the reconstructed signals in multichannel compression [9], [13].

\subsection{Telemedicine}

To demonstrate the efficiency of the WSAT method, the MITBIH arrhythmia database is selected. The sample values are in the range of -1024 to 1023 , and the sample resolution is set as $11 \mathrm{bits} / \mathrm{sample}$. The biorthogonal 9/7 tap filters are chosen, and a 5-level wavelet decomposition is applied. It ought to be noted that entropy coding has not been used for presented results.

As recommended by the American Heart Association for routine visual readings of compressed and reconstructed ECG signals, the PRD value may not exceed 5\% [14].

The proposed method was tested using 14 records from the MIT-BIH arrhythmia database (1-min length of data in record numbers 100, 104, 107, 111, 112, 115, 116, 117, 118, 119, 121, 213,214 , and 231) and the sampling rate is 360 samples/sec [15]. The results for dual-channel ECG are shown in Figure 2. 
As recommended in [14], to do final analysis and diagnostic interpretation of the retrieved ECG, PRD value may not exceed 5\%. In Figure 2, the APRD values for the selected records are presented corresponding to certain CDRs. If the average of APRD values for each graph is calculated (i.e. average of APRDs at a certain CDR), it is seen that the average of APRDs is less than $5 \%$ per two channels. Therefore, diagnostic features of the reconstructed data have been preserved.

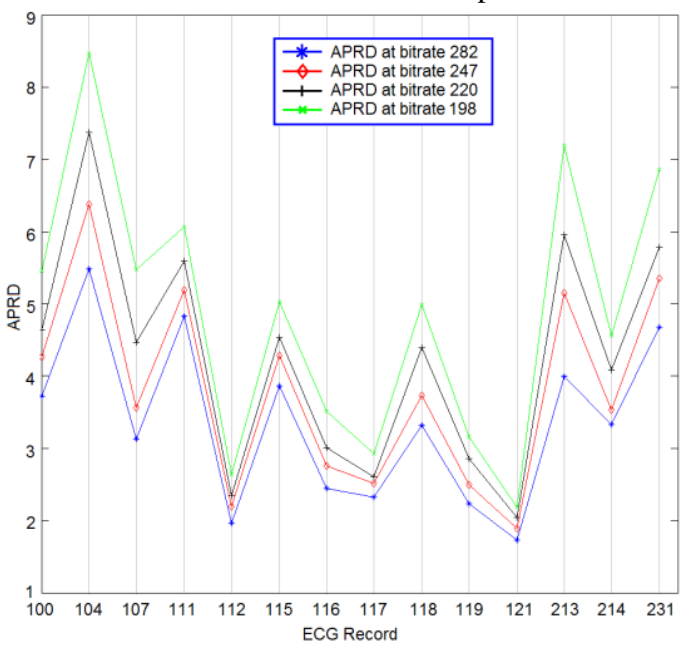

Figure 2. APRD values for selected records corresponding to certain bitrates

In order to illustrate the effect of proposed compression on the reconstructed signal, the signal from record 117 and its reconstructed signal are shown in Figure 3. For the data shown in the figure, the PRD is $1.72 \%$ for channel 1 , and $2.73 \%$ for channel 2 at bit rate 300 bps per two channels (the sampling rate is 360 samples/sec).
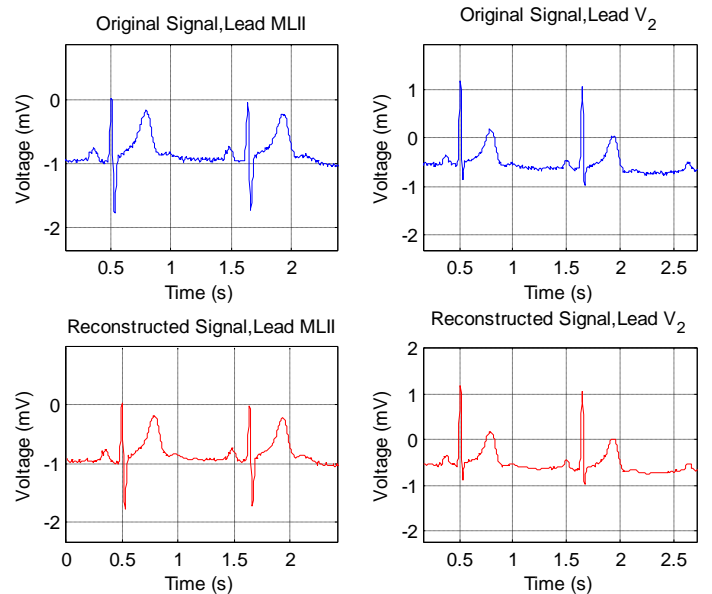

Figure 3. The original and reconstructed dual-channel ECG of record 117

Reported results of various multichannel (MC) ECG compression algorithms which are presented in literature [16][21] are considered under different simulation conditions, such as different database, sampling rate, sample resolution, and noise level. Therefore various algorithms can be difficultly compared.

The MC-LTP algorithm which is the multichannel case of longterm prediction (LTP) algorithm [22], was reported in [18] for MC ECG compression. In [18], the APRD value of $7.1 \%$ with bit rate 154 bps per channel was reported for the record of 233 which resampled at $250 \mathrm{~Hz}$. The APRD value of the WSAT is $2.44 \%$ for the same record and CDR. The rate-distortion curves

for records 111, 116, 212, 213, 219, 231, and 233 are provided in Figure 4 and compared with reported results in [18]. The superiority of the WSAT is clearly shown from Figure 4.

The multirate algorithm based on DCT transformation, was provided in [17]. It is very difficult to compare results of the WSAT with the results of the multirate algorithm, but the results reported here seem to be inferior to the WSAT.

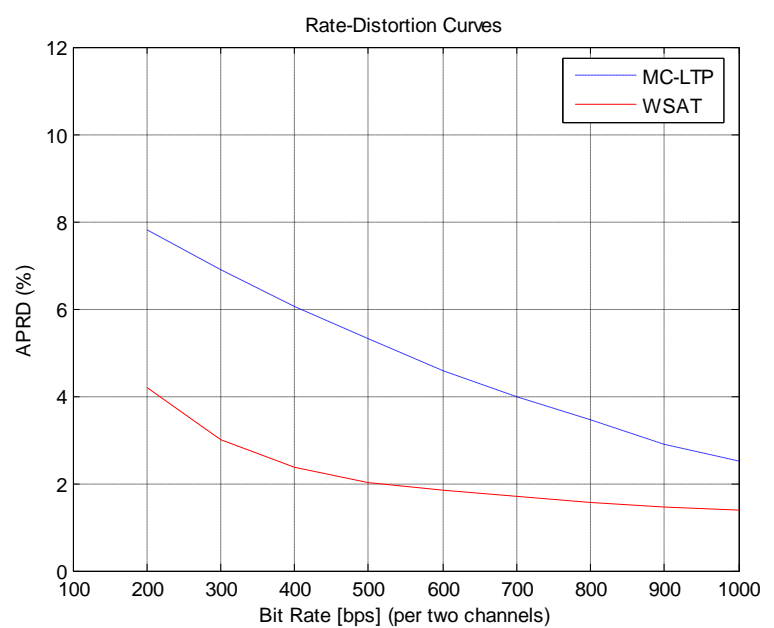

Figure 4. Rate-distortion curves with APRD

The vector quantization (VQ) algorithm based on the AZTEC algorithm was presented in [16] for MC ECG data compression. In comparison with VQ-coded m-AZTEC algorithm, the results of the WSAT are completely better than the reported results of this algorithm.

Miaou et al. proposed a multichannel version of adaptive vector quantization (AVQ) [19]. They tested all 48 records of ECG data with dual-channel at sampling rate of 360 samples/sec and sample resolution of $11 \mathrm{bits} / \mathrm{sample}$. They reported the APRD value of $9.8 \%$ of 48 records for channel 1 and $17.8 \%$ for channel 2 (APRD for both channels is $13.8 \%$ ) at average bit rate 238.2 bps per channel. The total APRD (APRD for both channels) value of the proposed technique is $2.8 \%$ for the same records and CDR.

\subsection{Meteorology}

To show the efficiency of the proposed method on compression of climate data, the data from the Nevada climate change database [23] is utilized. The method is tested on two datasets.

\subsubsection{Dataset I}

The 4-month air temperature data (10-meter height) from the research sites including Snake range Sagebrush (East), Sheep range Pinyon-Juniper, Sheep range Montane, Sheep range Mojave desert shrub, Sheep range Blackbrush are considered. The temperature data consists of maximum, minimum and average values. The sample resolution is set as $12 \mathrm{bits} / \mathrm{sample}$, and samples are collected every 10 mins. The Daubechies 4 wavelets are chosen [24], and a 5-level wavelet decomposition is applied. The average PRD results at different CR's are presented in Table I.

Figure 5 shows the original and reconstructed signal of average, minimum, and maximum values of air temperature (10-meter height) from the Sheep range Mojave desert shrub site between $16^{\text {th }}$ July-21 $1^{\text {st }}$ July 2013 . The Compression ratio is 20 per three channels, and APRD is $1.91 \%$. 


\subsubsection{Dataset II}

The 4-month relative humidity (2-meter height), air temperature (2-meter height) and wind velocity (10-meter height) data from the research sites including Snake range Subalpine (West), Snake range salt desert shrub (East), Snake range Sagebrush (East), Snake range Sagebrush (West), Snake range PinyonJuniper are considered. The sample resolution is set as 12 bits/sample, and samples are collected every 10 mins. The average PRD results at different CR's per 3 channels are illustrated in Figure 6.

Table1. The results of the WSAT technique for first dataset; CR per three channels

\begin{tabular}{|c|c|c|c|c|c|c|}
\hline Research Site & $\begin{array}{c}\text { APRD at } \\
\text { CR=30 }\end{array}$ & $\begin{array}{c}\text { APRD at } \\
\text { CR=20 }\end{array}$ & $\begin{array}{c}\text { APRD at } \\
\text { CR=15 }\end{array}$ & $\begin{array}{c}\text { APRD at } \\
\text { CR=10 }\end{array}$ & $\begin{array}{c}\text { APRD at } \\
\text { CR=5 }\end{array}$ & $\begin{array}{c}\text { APRD at } \\
\text { CR=3 }\end{array}$ \\
\hline $\begin{array}{c}\text { Snake Range Sagebrush } \\
\text { (East) }\end{array}$ & 3.06 & 2.28 & 1.96 & 1.42 & 0.74 & 0.29 \\
\hline Sheep Range Pinyon-Juniper & 2.65 & 1.93 & 1.54 & 1.19 & 0.62 & 0.24 \\
\hline Sheep range Montane & 3.26 & 2.31 & 1.87 & 1.46 & 0.78 & 0.31 \\
\hline Sheep Range Mojave desert shrub & 2.56 & 1.91 & 1.53 & 0.99 & 0.46 & 0.19 \\
\hline Sheep Range Blackbrush & 3.07 & 2.07 & 1.65 & 1.2 & 0.6 & 0.24 \\
\hline Total Average & 2.92 & 2.1 & 1.71 & 1.25 & 0.64 & 0.25 \\
\hline
\end{tabular}
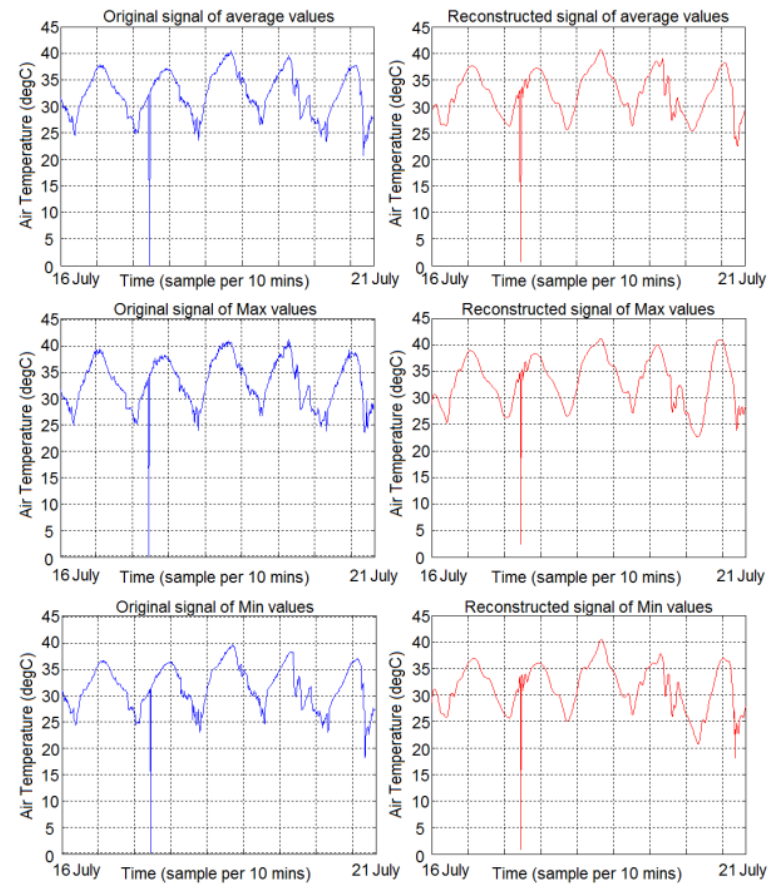

Figure 5. The original and reconstructed signal of average, minimum, and maximum values of air temperature; Values of air temperature from 12 a.m., 16 July to 12 a.m., 21 July 2013.

\section{CONCLUSION}

In this paper, the wavelet subbands arranging technique was proposed to simultaneously compress multichannel data. In comparison with famous multichannel compression methods such as adaptive vector quantization [19], multichannel case of long-term prediction (MC-LTP) [22], the method has better performance at the same PRD due to the appropriate rearrangement of wavelet subbands of input channels, and the efficient evaluation of the wavelet coefficients; appropriate rearrangement of the wavelet subbands provides convenient parent-offspring relations among coefficients of wavelet subbands at a hierarchical structure. In addition, the multichannel compression can be stopped as soon as the desired bit rate or quality requirement is achieved. It means a user has exact control on bit rate. Finally, with the progressive coding capability of the proposed method, the signal quality can be gradually improved as the bit rate increases. The proposed method also reduces the number of stored or transmitted bits in a bit stream. Whereas the proposed method gradually improves the quality of reconstructed signals, if redundant message bits are decreased, much important data will be sent at a certain bit rate. This fact will extremely affect results if a decrement in the bits leads to increase transmission of bits relating to coefficients of low frequency subbands. Consequently, the reconstruction error in the decoder side will be decreased. The method is an attractive choice for applications in which real time processing is crucial. In fact, the capability of more convenient real-time analysis of data can be provided for specialists. The algorithm was applied on selected records from the MIT-BIH arrhythmia database, and selected data from Nevada climate change data base. The experimental results confirmed the proposed technique has a good performance in quality versus CDR.

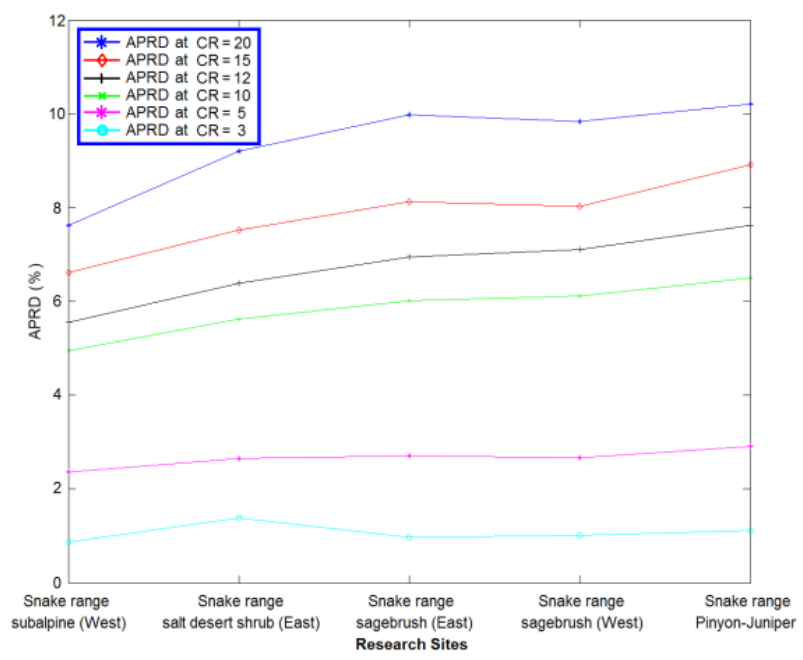

Figure 6. APRD values of relative humidity, air temperature, and wind velocity from selected research sites corresponding to certain CR's 


\section{AKNOWLEDGEMENT}

This work is supported (in part) by the Defense Threat Reduction Agency, Basic Research Award \# HDTRA1-12-10033, and the National Science Foundation (NSF) award \#EPSIIA-1301726. Any findings, conclusions, or recommendations expressed in the material are those of the author(s) and do not necessarily reflect the views of NSF.

\section{REFERENCES}

[1] Thakor N. V. "Ambulatory arrhythmia monitoring: From holter monitors to automatic implantable defibrillators," IEEE Transaction on Biomedical Engineering, vol.31, 1984, pp.770-778.

[2] Chan H. L., Siao Y. C., et al. "Wavelet-based ECG compression by bit-field preserving and running length encoding," Computer Methods and Programs in Biomedicine, Elsevier, vol. 90, 2008, pp.1-8.

[3] Zigel Y., Cohen A., and Katz A. "ECG signal compression using analysis by synthesis coding," IEEE Transaction on Biomedical Engineering, vol. 47, Oct. 2000, pp.13081315 .

[4] Miaou S. G., and Chao S. N., "Wavelet-based lossy to lossless ECG compression in a unified vector quantization framework," IEEE Trans. Biomed. Eng., vol. 52, Mar. 2005, pp. 539-543.

[5] Hilton M. L., "Wavelet and wavelet packet compression of electrocardiograms," IEEE Trans. Biomed. Eng, vol. 44, May 1997, pp. 394-402.

[6] Lu Z., Kim D. Y., et al., "Wavelet compression of ECG signals by the set partitioning in hierarchical trees algorithm," IEEE Trans. Biomed. Eng, vol. 47, July 2000, pp. 849-856.

[7] Thakor N. V., Webster J. G., and Tompkins W. J., "Estimation of QRS complex power spectra for design of a QRS filter," IEEE Trans. Biomed. Eng., vol.31, Nov. 1984, pp. 702-706.

[8] Cohen A., Daubechies I., and Feauveau J. C., "Biorthogonal bases of compactly supported wavelets," Communications on Pure and Applied Mathematics, vol. 45, 1992, pp. 485-560.

[9] Sharifahmadian E., "Wavelet compression of multichannel ECG data by enhanced set partitioning in hierarchical trees algorithm," $28^{\text {th }}$ IEEE EMBS Annual International Conference, New York City, USA, Sept. 2006, pp. 52385243.

[10] Mallat S. G., "A theory for multiresolution signal decomposition: The wavelet representation," IEEE Transaction on Pattern analysis and machine intelligence, vol. 11, July 1989, pp. 674-693.
[11] Nave G., and Cohen A., "ECG compression using longterm prediction", IEEE Trans. Biomed. Eng., vol. 40, Sept. 1993, pp. 877-885.

[12] Miaou S. G., and Yen H. L., "Quality driven gold washing adaptive vector quantization and its application to ECG data compression", IEEE Trans. Biomed. Eng., vol.47, Feb. 2000, pp. 209-218.

[13] Jalaleddine S. M. S., Hutchens C., et al., "ECG data compression techniques-A unified approach," IEEE Trans. Biomed. Eng, vol. BME-37, Apr.1990, pp. 329-343.

[14] Bailey J. J., Berson A. S., et al. "Recommendations for standardization and specifications in automated electrocardiography-Bandwidth and digital signal processing," Circulation, vol. 81, Feb. 1990, pp. 730-739.

[15] http://www.physionet.org/physiobank/database/mitdb/

[16] Mammen C. P., and Ramamurthi B., "Vector quantization for compression of multichannel ECG," IEEE Trans. Biomed. Eng., vol. 37, Sept. 1990, pp. 821-825.

[17] Cetin A. E., Koymen H., and Aydin M. C., "Multichannel ECG data compression by multirate signal processing and transform domain coding techniques," IEEE Trans. Biomed. Eng., vol. 40, May 1993, pp. 495-499.

[18] Cohen A., and Zigel Y., "Compression of multichannel ECG through multichannel long-term prediction," IEEE Eng. Med. Biol. Mag., vol. 16, no. 4, May 1998, pp. 109 115.

[19] Miaou S. G., and Yen H. L., "Multichannel ECG compression using multichannel adaptive vector quantization," Communications of IEEE Trans. Biomed. Eng., vol. 48, no. 10, Oct. 2001, pp. 1203-1207.

[20] Sharma L. N., Dandapat S., and Mahanta A., "Multichannel ECG data compression based on multiscale principal component analysis", IEEE Transactions on Information Technology in Biomedicine, Vol.16, No.4, July 2012, pp.730-736.

[21] Liu B., Zhang Z., et al., "Compression via compressive sensing: A low-power framework for the tele-monitoring of multi-channel physiological signals", IEEE Inter. Conf on Bioinformatics and Biomedicine (BIBM), China, Dec 2013, pp.9-12.

[22] Nave G., Cohen A., "ECG compression using long-term prediction,” IEEE Trans. Biomed. Eng., vol. 40, Sept. 1993, pp. 877-885.

[23] http://sensor.nevada.edu/NCCP/Default.aspx

[24] Abdul Karim S. A., Abdul Karim B., et al., "Compression of temperature data by using Daubechies wavelets," Proc. $2^{\text {nd }}$ International Conference on Mathematical Sciences (ICMS2), 2010, pp. 726-734. 